DOI 10.4171/JEMS/190

U. Kohlenbach · L. Leuştean

\title{
Asymptotically nonexpansive mappings in uniformly convex hyperbolic spaces
}

\author{
Dedicated to Professor Georg Kreisel on the occasion of his 85th birthday
}

Received August 8, 2007 and in revised form March 26, 2008

\begin{abstract}
This paper provides a fixed point theorem for asymptotically nonexpansive mappings in uniformly convex hyperbolic spaces as well as new effective results on the Krasnosel'skiı̌-Mann iterations of such mappings. The latter were found using methods from logic and the paper continues a case study in the general program of extracting effective data from prima-facie ineffective proofs in the fixed point theory of such mappings.
\end{abstract}

Keywords. Proof mining, functionals of finite type, metric fixed point theory, asymptotically nonexpansive mappings, uniformly convex hyperbolic spaces, CAT(0)-spaces, Krasnosel'skiı̌-Mann iterations

\section{Introduction}

This paper provides a fixed point theorem for asymptotically nonexpansive mappings in uniformly convex hyperbolic spaces (Theorem 3.3) as well as new effective results on the Krasnosel'skiı̌-Mann iterations of such mappings (Theorem 3.5. The fixed point theorem generalizes corresponding theorems for uniformly convex normed spaces ([6]) and CAT(0)-spaces ([11]) while the effective bounds on the Krasnosel'skiī-Mann iterations generalize results from [16] for the normed case which were obtained using techniques from mathematical logic or, more specifically, a proof-theoretic method called (monotone) functional interpretation (see [12, 4]). In this respect the current paper continues a case study in the general program of 'proof mining' which is concerned with the extraction of effective uniform bounds from (prima-facie) ineffective proofs (see the discussion in Section 5 and [14] for a survey as well as [15]). Monotone functional interpretation systematically transforms any statement in a given proof into a new constructive version for which explicit bounds are provided. In the case of convergence statements (which this paper is about) this coincides with what recently has been advocated under the name

U. Kohlenbach: Department of Mathematics, Technische Universität Darmstadt, Schlossgartenstrasse 7, 64289 Darmstadt, Germany e-mail: kohlenbach@mathematik.tu-darmstadt.de

L. Leuştean: Department of Mathematics, Technische Universität Darmstadt, Schlossgartenstrasse 7, 64289 Darmstadt, Germany, and Institute of Mathematics "Simion Stoilow" of the Romanian Academy, Calea Griviţei 21, P.O. Box 1-462, Bucureşti, Romania; e-mail: leustean@mathematik.tu-darmstadt.de

Mathematics Subject Classification (2010): 03F10, 47H10, 47H09 
'metastability' or 'finite convergence' in an essay posted by T. Tao ([28], see also [29]). Thus the paper can also be seen as an instance of 'hard analysis' as proposed by Tao.

Since the fundamental paper [6], the class of asymptotically nonexpansive mappings has been much studied in fixed point theory. Let $(X, d)$ be a metric space. A function $T: X \rightarrow X$ is called asymptotically nonexpansive if for some sequence $\left(k_{n}\right)$ in $[0, \infty)$ with $\lim _{n \rightarrow \infty} k_{n}=0$ one has

$$
d\left(T^{n} x, T^{n} y\right) \leq\left(1+k_{n}\right) d(x, y), \quad \forall n \in \mathbb{N}, \forall x, y \in X .
$$

Asymptotically nonexpansive mappings have been studied mostly in the context of uniformly convex normed spaces (in fact, for general normed spaces it is even open whether asymptotically nonexpansive selfmappings of bounded, closed, convex subsets have approximate fixed points, see [5]). One typical result is the following theorem which is proved in [16, Corollary 8] (as a corollary of a quantitative result) but essentially is contained already in [23, 24, 25, 21]:

Theorem 1.1. Let $(X,\|\cdot\|)$ be a uniformly convex normed space, $C \subseteq X$ a convex subset and $T: C \rightarrow C$ an asymptotically nonexpansive mapping with sequence $\left(k_{n}\right)$ in $[0, \infty)$ satisfying $\sum_{i=0}^{\infty} k_{i}<\infty$. Let $\left(\lambda_{n}\right)$ be a sequence in $[a, b]$ for $0<a<b<1$ and define the Krasnosel'skiu-Mann iteration of $T$ starting from $x \in X$ by

$$
x_{0}:=x, \quad x_{n+1}:=\left(1-\lambda_{n}\right) x_{n}+\lambda_{n} T^{n}\left(x_{n}\right) .
$$

If $T$ has a fixed point, then $\left\|x_{n}-T\left(x_{n}\right)\right\| \rightarrow 0$ as $n \rightarrow \infty$.

While there does not seem to exist a computable rate of convergence in this case (in [16] it is shown that the proof even holds for asymptotically weakly-quasi nonexpansive functions for which one can show that no uniform effective rate exists), general logical metatheorems from [13, 4] guarantee (see also Section 5 below) an effective uniform bound on the so-called no-counterexample interpretation (see Kreisel [17]) of the convergence, or - to use Tao's [28, 29] terminology—on the metastability of $\left(\left\|x_{n}-T\left(x_{n}\right)\right\|\right)$, i.e. on

$$
\forall \varepsilon>0 \forall g: \mathbb{N} \rightarrow \mathbb{N} \exists N \in \mathbb{N} \forall m \in[N, N+g(N)]\left(\left\|x_{m}-T\left(x_{m}\right)\right\|<\varepsilon\right),
$$

which (ineffectively) is equivalent to the regular formulation of convergence towards 0 . Here $[n, n+m]:=\{n, n+1, \ldots, n+m\}$.

The proof analyzed in [16] uses a lemma from [20]:

Lemma 1.2 ([20]). Let $\left(a_{n}\right),\left(b_{n}\right),\left(c_{n}\right)$ be sequences in $\mathbb{R}_{+}$such that $\sum b_{n}$ and $\sum c_{n}$ are bounded and

$$
\forall n \in \mathbb{N}\left(a_{n+1} \leq\left(1+b_{n}\right) a_{n}+c_{n}\right) .
$$

Then $\left(a_{n}\right)$ is convergent.

The results in [16] were obtained by transforming a proof of $\left\|x_{n}-T\left(x_{n}\right)\right\| \rightarrow 0$ based on Lemma 1.2 into a proof of $(*)$ together with an explicit effective bound for $(*)$ using a corresponding effective bound for the 'metastability' version of Lemma 1.2 (see also Proposition 6.4 below) which constitutes a generalization of Tao's finite convergence principle from [28]. 
In this paper we take the proofs from [16] as our point of departure and generalize the results to uniformly convex hyperbolic spaces (see the next section). This, in particular, covers the important class of CAT(0)-spaces (in the sense of Gromov) and, a fortiori, $\mathbb{R}$-trees in the sense of Tits. For CAT(0)-spaces we get a quadratic bound on the approximate fixed point property of $\left(x_{n}\right)$ (see Corollary 3.11).

\section{Hyperbolic spaces-definitions and properties}

One can find in the literature different notions of 'hyperbolic space' [10, 7, 8, 22]. We work in the setting of hyperbolic spaces as introduced by the first author [13], which are slightly more restrictive than the spaces of hyperbolic type in the sense of GoebelKirk [7], but more general than the hyperbolic spaces in the sense of Reich-Shafrir [22].

A hyperbolic space $(X, d, W)$ is a metric space $(X, d)$ together with a convexity mapping $W: X \times X \times[0,1] \rightarrow X$ satisfying

(W1) $d(z, W(x, y, \lambda)) \leq(1-\lambda) d(z, x)+\lambda d(z, y)$,

(W2) $d(W(x, y, \lambda), W(x, y, \tilde{\lambda}))=|\lambda-\tilde{\lambda}| \cdot d(x, y)$,

(W3) $W(x, y, \lambda)=W(y, x, 1-\lambda)$,

(W4) $d(W(x, z, \lambda), W(y, w, \lambda)) \leq(1-\lambda) d(x, y)+\lambda d(z, w)$.

The convexity mapping $W$ was first considered by Takahashi in [27], where a triple $(X, d, W)$ satisfying $(\mathrm{W} 1)$ is called a convex metric space.

The class of hyperbolic spaces includes normed spaces and convex subsets thereof, the Hilbert ball [8] as well as CAT(0)-spaces in the sense of Gromov (see [2] for a detailed treatment).

If $x, y \in X$ and $\lambda \in[0,1]$ then we use the notation $(1-\lambda) x \oplus \lambda y$ for $W(x, y, \lambda)$. It is easy to see that for any $x, y \in X$ and any $\lambda \in[0,1]$,

$$
d(x,(1-\lambda) x \oplus \lambda y)=\lambda d(x, y) \quad \text { and } \quad d(y,(1-\lambda) x \oplus \lambda y)=(1-\lambda) d(x, y) .
$$

We shall denote by $[x, y]$ the set $\{(1-\lambda) x \oplus \lambda y: \lambda \in[0,1]\}$. A nonempty subset $C \subseteq X$ is convex if $[x, y] \in C$ for all $x, y \in C$.

For any $x \in X, r>0$, the open (respectively closed) ball with center $x$ and radius $r$ is denoted by $U(x, r)$ (respectively $\bar{U}(x, r))$. It is easy to see that open balls and closed balls are convex. Moreover, using (W4), we see that the closure of a convex subset of a hyperbolic space is again convex.

One of the most important classes of Banach spaces are the uniformly convex ones, introduced by Clarkson in the 30's [3]. Following [8, p. 105], we can define uniform convexity for hyperbolic spaces too.

A hyperbolic space $(X, d, W)$ is uniformly convex [18] if for any $r>0$ and any $\varepsilon \in(0,2]$ there exists $\theta \in(0,1]$ such that for all $a, x, y \in X$,

$$
\left.\begin{array}{l}
d(x, a) \leq r \\
d(y, a) \leq r \\
d(x, y) \geq \varepsilon r
\end{array}\right\} \Rightarrow d\left(\frac{1}{2} x \oplus \frac{1}{2} y, a\right) \leq(1-\theta) r
$$


A mapping $\eta:(0, \infty) \times(0,2] \rightarrow(0,1]$ providing such a $\theta:=\eta(r, \varepsilon)$ for given $r>0$ and $\varepsilon \in(0,2]$ is called a modulus of uniform convexity.

In the following, $(X, d, W)$ is a uniformly convex hyperbolic space and $\eta$ is a modulus of uniform convexity.

Lemma 2.1. Let $r>0, \varepsilon \in(0,2]$ and $a, x, y \in X$ be such that $d(x, a) \leq r, d(y, a) \leq r$, $d(x, y) \geq \varepsilon r$. Then for any $\lambda \in[0,1]$,

1. $d((1-\lambda) x \oplus \lambda y, a) \leq(1-2 \lambda(1-\lambda) \eta(r, \varepsilon)) r$;

2. for any $\psi \in(0,2]$ such that $\psi \leq \varepsilon$,

$$
d((1-\lambda) x \oplus \lambda y, a) \leq(1-2 \lambda(1-\lambda) \eta(r, \psi)) r ;
$$

3. for any $s \geq r$,

$$
d((1-\lambda) x \oplus \lambda y, a) \leq(1-2 \lambda(1-\lambda) \eta(s, \varepsilon r / s)) s .
$$

Proof. 1. See [18, Lemma 7].

2. Note that $d(x, y) \geq \varepsilon r \geq \psi r$ and apply item 1

3. Since $d(x, a), d(y, a) \leq r \leq s, d(x, y) \geq \varepsilon r=(\varepsilon r / s) s$ and $0<\varepsilon r / s \leq \varepsilon \leq 2$, the conclusion follows again by an application of item 1 .

We say that $\eta$ is monotone if it decreases with $r$ (for a fixed $\epsilon$ ). It turns out that $\operatorname{CAT}(0)$ spaces are uniformly convex hyperbolic spaces having a monotone modulus of uniform convexity, quadratic in $\varepsilon: \eta(r, \varepsilon)=\varepsilon^{2} / 8$. We refer to [18] for details.

The following proposition is one of the main ingredients in the proof of Theorem 3.3 Its proof is similar to the one of the corresponding result for uniformly convex Banach spaces (see, for example, [8, Theorem 2.1]).

Proposition 2.2. Let $(X, d, W)$ be a complete uniformly convex hyperbolic space with a monotone modulus of uniform convexity $\eta$. The intersection of any decreasing sequence of nonempty bounded closed convex subsets of $X$ is nonempty.

Proof. Let $\left(C_{n}\right)_{n \geq 1}$ be a decreasing sequence of nonempty bounded closed convex subsets of $X$ and let $x \in X$ be arbitrary. If $x \in C_{n}$ for all $n \in \mathbb{N}$, then $\bigcap_{n \geq 1} C_{n} \neq \emptyset$. Assume that there exists $N \in \mathbb{N}$ such that $x \notin C_{N}$, so that $d\left(x, C_{N}\right)>0$, since $C_{N}$ is closed. If $r_{n}:=d\left(x, C_{n}\right)$, then $\left(r_{n}\right)$ is an increasing sequence of nonnegative reals, bounded from above by $d(x, a)+\operatorname{diam}\left(C_{1}\right)$, where $a \in C_{1}$. It follows that $r:=\lim r_{n}=\sup r_{n} \geq$ $r_{N}>0$.

Define $D_{n}:=C_{n} \cap \bar{U}(x, r+1 / n)$. Then it is easy to see that $\left(D_{n}\right)$ is a decreasing sequence of nonempty closed subsets of $X$. Let $d_{n}:=\operatorname{diam}\left(D_{n}\right)$ and $0 \leq d:=\lim d_{n}=$ $\inf d_{n}$.

Assume that $d>0$. Let $K \in \mathbb{N}$ be such that $1 / K \leq d / 2$. For any $n \geq K$, there exist $x_{n}, y_{n} \in D_{n}$ such that $d\left(x_{n}, y_{n}\right) \geq d_{n}-1 / n \geq d-1 / n \geq d / 2$.

Since $d\left(x_{n}, x\right), d\left(y_{n}, x\right) \leq r+1 / n$ and

$$
d\left(x_{n}, y_{n}\right) \geq \frac{d}{2} \geq\left(r+\frac{1}{n}\right) \cdot \frac{d}{2(r+1)}, \quad \frac{d}{2(r+1)} \leq 1,
$$


we see that for all $n \geq K$,

$$
\begin{aligned}
r_{n} & \leq d\left(\frac{1}{2} x_{n} \oplus \frac{1}{2} y_{n}, x\right) \leq\left(1-\eta\left(r+\frac{1}{n}, \frac{d}{2(r+1)}\right)\right) \cdot\left(r+\frac{1}{n}\right) \\
& \leq\left(1-\eta\left(r+1, \frac{d}{2(r+1)}\right)\right) \cdot\left(r+\frac{1}{n}\right),
\end{aligned}
$$

(since $r+1 / n \leq r+1$ and $\eta$ is monotone).

Thus, letting $n \rightarrow \infty$ yields

$$
r \leq\left(1-\eta\left(r+1, \frac{d}{2(r+1)}\right)\right) \cdot r<r,
$$

a contradiction. It follows that we must have $d=0$. This and the completeness of $X$ imply that $\bigcap_{n \geq 1} D_{n} \neq \emptyset$, hence $\bigcap_{n \geq 1} C_{n} \neq \emptyset$.

\section{Main results}

The notion of nonexpansive mapping can be introduced in the very general setting of metric spaces. If $(X, d)$ is a metric space, and $C \subseteq X$ a nonempty subset, then a mapping $T: C \rightarrow C$ is called nonexpansive if for all $x, y \in C$,

$$
d(T x, T y) \leq d(x, y)
$$

Asymptotically nonexpansive mappings were introduced by Goebel and Kirk [6] as a generalization of the nonexpansive ones. A function $T: C \rightarrow C$ is said to be asymptotically nonexpansive with sequence $\left(k_{n}\right)_{n \geq 0}$ in $[0, \infty)$ if $\lim _{n \rightarrow \infty} k_{n}=0$ and

$$
d\left(T^{n} x, T^{n} y\right) \leq\left(1+k_{n}\right) d(x, y), \quad \forall n \in \mathbb{N}, \forall x, y \in C .
$$

Fix $(T)$ denotes the set of fixed points of $T$ and for any $\varepsilon>0, \operatorname{Fix}_{\varepsilon}(T)$ denotes the set of $\varepsilon$-fixed points, that is, points $x \in C$ such that $d(x, T x)<\varepsilon$.

We say that $C$ has the fixed point property (FPP) for asymptotically nonexpansive mappings if $\operatorname{Fix}(T) \neq \varnothing$ for any asymptotically nonexpansive mapping $T: C \rightarrow C$. Moreover, $C$ has the approximate fixed point property (AFPP) for asymptotically nonexpansive mappings if $\operatorname{Fix}_{\varepsilon}(T) \neq \emptyset$ for any asymptotically nonexpansive mapping $T$ : $C \rightarrow C$ and any $\varepsilon>0$.

Goebel and Kirk proved the following generalization of the famous Browder-Goehde -Kirk fixed point theorem for nonexpansive mappings.

Theorem 3.1 ([6, Theorem 1]). Nonempty closed convex and bounded subsets of uniformly convex Banach spaces have the FPP for asymptotically nonexpansive mappings.

In 2004, Kirk obtained a similar result for CAT(0)-spaces. 
Theorem 3.2 ([11, Theorem 28]). Nonempty closed convex and bounded subsets of complete CAT $(0)$-spaces have the FPP for asymptotically nonexpansive mappings.

Kirk proved Theorem 3.2 using nonstandard methods, inspired by Khamsi's proof that bounded hyperconvex metric spaces have the AFPP for asymptotically nonexpansive mappings [9].

The first main result of this paper is a generalization of Theorem 3.1 to uniformly convex hyperbolic spaces with monotone modulus of uniform convexity.

Theorem 3.3. Let $(X, d, W)$ be a complete uniformly convex hyperbolic space having a monotone modulus of uniform convexity. Then any nonempty closed convex and bounded subset of $X$ has the FPP for asymptotically nonexpansive mappings.

Our proof follows closely Goebel and Kirk's proof of Theorem 3.1 and we present the details in Section 4 As a consequence, we also obtain an elementary proof of Theorem 3.2

In fact, as already pointed out for uniformly convex normed spaces in [16], the proof of the FPP can be transformed into an elementary proof of the AFPP, which does not need the completeness of $X$ or the closedness of $C$.

Proposition 3.4. Let $(X, d, W)$ be a uniformly convex hyperbolic space having a monotone modulus of uniform convexity. Then any nonempty convex and bounded subset of $X$ has the AFPP for asymptotically nonexpansive mappings.

Proof. The proof of [16, Lemma 21] generalizes easily to our setting.

The main part of the paper will be devoted to getting a quantitative version of an asymptotic regularity theorem for the Krasnosel'skiǐ-Mann iterations of asymptotically nonexpansive mappings.

Let $(X, d, W)$ be a hyperbolic space, $C \subseteq X$ a nonempty convex subset of $X$ and $T: C \rightarrow C$ an asymptotically nonexpansive mapping.

For asymptotically nonexpansive mappings, the Krasnosel'skil-Mann iteration starting from $x \in C$ is defined by

$$
x_{0}:=x, \quad x_{n+1}:=\left(1-\lambda_{n}\right) x_{n} \oplus \lambda_{n} T^{n} x_{n},
$$

where $\left(\lambda_{n}\right)$ is a sequence in $[0,1]$.

Following [1], we say that $T$ is $\lambda_{n}$-asymptotically regular if for all $x \in C$,

$$
\lim _{n \rightarrow \infty} d\left(x_{n}, T x_{n}\right)=0
$$

The second main result of the paper is the following theorem, generalizing to uniformly convex hyperbolic spaces a similar result obtained for uniformly convex normed spaces by the first author and Lambov [16]. 
Theorem 3.5. Let $(X, d, W)$ be a uniformly convex hyperbolic space with a monotone modulus of uniform convexity $\eta, C$ be a nonempty convex subset of $X$ and $T: C \rightarrow C$ be asymptotically nonexpansive with sequence $\left(k_{n}\right)$. Assume that $K \geq 0$ is such that $\sum_{n=0}^{\infty} k_{n} \leq K$ and that $L \in \mathbb{N}$ with $L \geq 2$ is such that $1 / L \leq \lambda_{n} \leq 1-1 / L$ for all $n \in \mathbb{N}$. Let $x \in C$ and $b>0$ be such that for any $\delta>0$ there is $p \in C$ with

$$
d(x, p) \leq b \wedge d(T p, p) \leq \delta .
$$

Then for all $\varepsilon \in(0,1]$ and for all $g: \mathbb{N} \rightarrow \mathbb{N}$,

$$
\exists N \leq \Phi(K, L, b, \eta, \varepsilon, g) \forall m \in[N, N+g(N)]\left(d\left(x_{m}, T x_{m}\right)<\varepsilon\right),
$$

where

$$
\begin{aligned}
& \Phi(K, L, b, \eta, \varepsilon, g):=h^{M}(0), \quad h(n):=g(n+1)+n+2, \\
& M:=\lceil 3(5 K D+D+11 / 2) / \theta\rceil, \quad D:=e^{K}(b+2), \\
& \theta:=\frac{\varepsilon}{L^{2} f(K)} \cdot \eta\left((1+K) D+1, \frac{\varepsilon}{f(K)((1+K) D+1)}\right), \\
& f(K):=2\left(1+(1+K)^{2}(2+K)\right) .
\end{aligned}
$$

Moreover, $N=h^{i}(0)+1$ for some $i<M$.

We shall give the proof of the above theorem in the last section of our paper. As we shall explain in detail in Section 5, the extractability of the bound $\Phi$ is guaranteed by a general logical metatheorem. Moreover, this theorem allows us to conclude that $\lim d\left(x_{n}, T x_{n}\right)$ $=0$, assuming the existence of approximate fixed points in some neighborhood of the starting point $x \in C$ (see the discussion on the Herbrand normal form in Section 5 .

Remark 3.6. By an inspection of its proof, it is easy to see that the above theorem remains true if we weaken the hypotheses on $\left(k_{n}\right)$ and $\left(\lambda_{n}\right)$. In fact, it is enough to require that $\sum_{n=0}^{\Phi} k_{n} \leq K$ and $1 / L \leq \lambda_{n} \leq 1-1 / L$ for all $n \leq \Phi$. Note that once the hypotheses are weakened one must quantify them over $\varepsilon$ and $g$ since $\Phi$ depends on these.

Remark 3.7. Assume, moreover, that $\eta(r, \varepsilon)$ can be written as $\eta(r, \varepsilon)=\varepsilon \cdot \tilde{\eta}(r, \varepsilon)$ where $\tilde{\eta}$ increases with $\varepsilon$ (for a fixed $r$ ). Then we can replace $\eta$ with $\tilde{\eta}$ in the bound $\Phi(K, L, b, \eta, \varepsilon, g)$.

Proof. Define

$$
\theta:=\frac{\varepsilon}{L^{2} f(K)} \cdot \tilde{\eta}\left((1+K) D+1, \frac{\varepsilon}{f(K)((1+K) D+1)}\right)
$$

and follow the proof of the theorem using Lemma 6.2 and 21) instead of Lemma 6.2 and 20 .

We now give some further corollaries. 
Theorem 3.8. Assume $(X, d, W), \eta, C, T: C \rightarrow C,\left(k_{n}\right), K,\left(\lambda_{n}\right), L$ are as in the hypotheses of Theorem 3.5. Let $x \in C$ and $b>0$ be such that for any $\delta>0$ there is $p \in C$ with

$$
d(x, p) \leq b \wedge d(T p, p) \leq \delta .
$$

Then $\lim d\left(x_{n}, T x_{n}\right)=0$ and, moreover,

$$
\forall \varepsilon \in(0,1] \exists N \leq \Phi(K, L, b, \eta, \varepsilon)\left(d\left(x_{N}, T x_{N}\right)<\varepsilon\right),
$$

where $\Phi(K, L, b, \eta, \varepsilon):=2 M$ and $M, D, \theta, f(K)$ are as in Theorem 3.5 .

Proof. Take $g(n) \equiv 0$ in Theorem 3.5

Corollary 3.9 (see also Theorem 5.2. Assume $(X, d, W), \eta, C, T: C \rightarrow C,\left(k_{n}\right)$, $K,\left(\lambda_{n}\right), L$ are as in the hypotheses of Theorem 3.5. If $\operatorname{Fix}(T) \neq \emptyset$, then $T$ is $\lambda_{n}$ asymptotically regular.

Proof. Let $\tilde{p}$ be a fixed point of $T$. For any $x \in C,(4)$ is satisfied with $b:=d(x, \tilde{p})$ and $p:=\tilde{p}$.

Corollary 3.10. Let $(X, d, W), \eta, C, T: C \rightarrow C,\left(k_{n}\right), K,\left(\lambda_{n}\right)$, L be as in the hypotheses of Theorem 3.5. Assume moreover that $C$ is bounded with finite diameter $d_{C}$. Then $T$ is $\lambda_{n}$-asymptotically regular, and the following holds for all $x \in C$ :

$$
\forall \varepsilon \in(0,1] \exists N \leq \Phi\left(K, L, d_{C}, \eta, \varepsilon\right)\left(d\left(x_{N}, T x_{N}\right)<\varepsilon\right),
$$

where $\Phi\left(K, L, d_{C}, \eta, \varepsilon\right)$ is defined as in Theorem 3.8 with $d_{C}$ replacing $b$.

Proof. If $C$ is bounded, then $C$ has the AFPP for asymptotically nonexpansive mappings by Proposition 3.4, so the condition (4) holds for all $x \in C$ with $d_{C}$ instead of $b$. Hence, we can conclude that $\lim d\left(x_{n}, T x_{n}\right)=0$ for all $x \in C$.

Thus, for bounded $C$, we get asymptotic regularity and an explicit approximate fixed point bound $\Phi\left(K, L, d_{C}, \eta, \varepsilon\right)$, which depends only on the error $\varepsilon$, on the modulus of uniform convexity $\eta$, on the diameter $d_{C}$ of $C$, on $\left(\lambda_{n}\right)$ via $L$ and on $\left(k_{n}\right)$ via $K$, but not on the nonexpansive mapping $T$, the starting point $x \in C$ of the iteration or other data related to $C$ and $X$. As we have pointed out in Section 2, CAT(0)-spaces are uniformly convex hyperbolic spaces with a 'nice' monotone modulus of uniform convexity $\eta(r, \varepsilon):=\varepsilon^{2} / 8$. Hence, as an immediate consequence of Corollary 3.10 and Remark 3.7 we get the following result.

Corollary 3.11. Let $X$ be a $C A T(0)$-space, $C$ be a nonempty convex bounded subset of $X$ with diameter $d_{C}$ and $T: C \rightarrow C$ be asymptotically nonexpansive with sequence $\left(k_{n}\right)$. Assume that $K \geq 0$ is such that $\sum_{n=0}^{\infty} k_{n} \leq K$ and that $L \in \mathbb{N}$ with $L \geq 2$ is such that $1 / L \leq \lambda_{n} \leq 1-1 / L$ for all $n \in \mathbb{N}$. Then $T$ is $\lambda_{n}$-asymptotically regular, and for all $x \in C$,

$$
\forall \varepsilon \in(0,1] \exists N \leq \Phi\left(K, L, d_{C}, \varepsilon\right)\left(d\left(x_{N}, T x_{N}\right)<\varepsilon\right),
$$


where

$$
\begin{aligned}
& \Phi\left(K, L, d_{C}, \varepsilon\right):=2 M \\
& M:=\left\lceil\frac{1}{\varepsilon^{2}} \cdot 24 L^{2}\left(5 K D+D+\frac{11}{2}\right)(f(K))^{3}((1+K) D+1)^{2}\right\rceil, \\
& D:=e^{K}\left(d_{C}+2\right), \quad f(K):=2\left(1+(1+K)^{2}(2+K)\right) .
\end{aligned}
$$

Hence, in the case of convex bounded subsets of CAT(0)-spaces, we get a quadratic (in $1 / \varepsilon$ ) approximate fixed point bound. We recall that for nonexpansive mappings, a quadratic rate of asymptotic regularity for the Krasnosel'skiı̌-Mann iterations was obtained by the second author [18].

\section{Proof of Theorem 3.3}

In this section, we give the proof of Theorem 3.3. As we have already pointed out, we generalize to our setting Goebel and Kirk's proof for uniformly convex Banach spaces.

Proof of Theorem 3.3 For any $y \in C$, set

$A_{y}:=\left\{a \in \mathbb{R}_{+} \mid\right.$there exist $x \in C$ and $k \in \mathbb{N}$ such that $d\left(T^{i} y, x\right) \leq a$ for all $\left.i \geq k\right\}$.

If $d_{C}$ is the diameter of $C$, then $d_{C} \in A_{y}$, hence $A_{y}$ is nonempty. Let $\alpha_{y}:=\inf A_{y}$. For any $\theta>0$ there exists $a_{\theta} \in A_{y}$ such that $a_{\theta}<\alpha_{y}+\theta$, so

$$
\exists x \in C \exists k \in \mathbb{N} \forall i \geq k\left(d\left(T^{i} y, x\right) \leq a_{\theta}<\alpha_{y}+\theta\right) .
$$

Obviously, $\alpha_{y} \geq 0$. We distinguish two cases:

Case 1: $\alpha_{y}=0$. Let $\varepsilon>0$. Applying [10) with $\theta:=\varepsilon / 2$, we get the existence of $x \in C$ and $k \in \mathbb{N}$ such that for all $m, n \geq k$,

$$
d\left(T^{m} y, T^{n} y\right) \leq d\left(T^{m} y, x\right)+d\left(T^{n} y, x\right)<\varepsilon / 2+\varepsilon / 2=\varepsilon,
$$

so the sequence $\left(T^{n} y\right)_{n \geq 1}$ is Cauchy, hence convergent to some $z \in C$. It is easy to see that $z$ is a fixed point of $T$.

Case 2: $\alpha_{y}>0$. For any $n \geq 1$, define

$$
C_{n}:=\bigcup_{k \geq 1} \bigcap_{i \geq k} \bar{U}\left(T^{i} y, \alpha_{y}+1 / n\right), \quad D_{n}:=\overline{C_{n}} \cap C .
$$

By 10 with $\theta:=1 / n$, there exist $x \in C$ and $k \geq 1$ such that $x \in \bigcap_{i \geq k} \bar{U}\left(T^{i} y, \alpha_{y}+1 / n\right)$, hence $D_{n}$ is nonempty. Moreover, $\left(D_{n}\right)_{n \geq 1}$ is a decreasing sequence of nonempty bounded closed convex subsets of $X$, hence we can apply Proposition 2.2 to deduce that

$$
D:=\bigcap_{n \geq 1} D_{n} \neq \varnothing
$$


Claim. For any $x \in D$ and $\theta>0$ there exists $K \in \mathbb{N}$ such that for all $i \geq K$,

$$
d\left(T^{i} y, x\right) \leq \alpha_{y}+\theta .
$$

Proof of claim. Let $x \in D, \theta>0$ and $N \in \mathbb{N}$ be such that $2 / N \leq \theta$. Since $x \in D$, we have $x \in \overline{C_{N}}$, so there exists a sequence $\left(x_{n}^{N}\right)_{n \geq 1}$ in $C_{N}$ such that $\lim x_{n}^{N}=x$. Let $P \geq 1$ be such that $d\left(x, x_{n}^{N}\right) \leq 1 / N$ for all $n \geq P$ and $K \geq 1$ such that $x_{P}^{N} \in$ $\bigcap_{i \geq K} \bar{U}\left(T^{i} y, \alpha_{y}+1 / N\right)$. It follows that for all $i \geq K$,

$$
d\left(T^{i} y, x\right) \leq d\left(T^{i} y, x_{P}^{N}\right)+d\left(x_{P}^{N}, x\right) \leq \alpha_{y}+1 / N+1 / N=\alpha_{y}+2 / N \leq \alpha_{y}+\theta .
$$

Thus, the claim is proved.

We shall prove that any point of $D$ is a fixed point of $T$. Let $x \in D$ and assume that $T x \neq x$. Then $\left(T^{n} x\right)$ does not converge to $x$, so there exists $\varepsilon>0$ such that

$$
\forall k \in \mathbb{N} \exists n \geq k\left(d\left(T^{n} x, x\right) \geq \varepsilon / 2\right) .
$$

We can of course assume that $\varepsilon \in(0,4]$. Then $\varepsilon /\left(2\left(\alpha_{y}+1\right)\right) \in(0,2]$ and there exists $\theta_{y} \in(0,1]$ such that

$$
1-\eta\left(\alpha_{y}+1, \frac{\varepsilon}{2\left(\alpha_{y}+1\right)}\right) \leq \frac{\alpha_{y}-\theta_{y}}{\alpha_{y}+\theta_{y}}
$$

Since $\lim \left(1+k_{n}\right)\left(\alpha_{y}+\theta_{y} / 2\right)=\alpha_{y}+\theta_{y} / 2<\alpha_{y}+\theta_{y}$, there exists $N_{0} \in \mathbb{N}$ such that

$$
\forall n \geq N_{0}\left(\left(1+k_{n}\right)\left(\alpha_{y}+\theta_{y} / 2\right)<\alpha_{y}+\theta_{y}\right) .
$$

By (13) with $\theta:=\theta_{y} / 2$, there exists $K \in \mathbb{N}$ such that

$$
\forall i \geq K\left(d\left(T^{i} y, x\right) \leq \alpha_{y}+\theta_{y} / 2\right) .
$$

Applying 14 with $k:=N_{0}$, we get $N \geq N_{0}$ such that

$$
d\left(T^{N} x, x\right) \geq \varepsilon / 2 .
$$

Let now $m \in \mathbb{N}$ be such that $m \geq N+K$. Then

$$
\begin{aligned}
d\left(T^{N} x, T^{m} y\right) & =d\left(T^{N} x, T^{N}\left(T^{m-N} y\right)\right) \leq\left(1+k_{N}\right) d\left(x, T^{m-N} y\right) \\
& \left.<\left(1+k_{N}\right)\left(\alpha_{y}+\theta_{y} / 2\right) \quad(\text { by } 17)\right) \\
& <\alpha_{y}+\theta_{y} \quad(\text { by } 16) .
\end{aligned}
$$

Hence,

$$
\begin{aligned}
d\left(T^{N} x, T^{m} y\right) & <\alpha_{y}+\theta_{y} \\
d\left(x, T^{m} y\right) & \leq \alpha_{y}+\theta_{y} / 2<\alpha_{y}+\theta_{y} \quad(\text { by }(17)) \\
d\left(x, T^{N} x\right) & \geq \varepsilon / 2 \quad\left(\text { by } \frac{\varepsilon}{18}\right) \\
& =\left(\alpha_{y}+\theta_{y}\right) \frac{\varepsilon}{2\left(\alpha_{y}+\theta_{y}\right)} \geq\left(\alpha_{y}+\theta_{y}\right) \frac{\varepsilon}{2\left(\alpha_{y}+1\right)} .
\end{aligned}
$$


Applying now the fact that $X$ is uniformly convex, we get

$$
d\left(\frac{1}{2} x \oplus \frac{1}{2} T^{N} x, T^{m} y\right) \leq\left(1-\eta\left(\alpha_{y}+\theta_{y}, \frac{\varepsilon}{2\left(\alpha_{y}+1\right)}\right)\right)\left(\alpha_{y}+\theta_{y}\right) .
$$

Since $\alpha_{y}+\theta_{y} \leq \alpha_{y}+1$ and $\eta$ is monotone,

$$
1-\eta\left(\alpha_{y}+\theta_{y}, \frac{\varepsilon}{2\left(\alpha_{y}+1\right)}\right) \leq 1-\eta\left(\alpha_{y}+1, \frac{\varepsilon}{2\left(\alpha_{y}+1\right)}\right) \leq \frac{\alpha_{y}-\theta_{y}}{\alpha_{y}+\theta_{y}}
$$

by 15 . Hence,

$$
d\left(\frac{1}{2} x \oplus \frac{1}{2} T^{N} x, T^{m} y\right) \leq \frac{\alpha_{y}-\theta_{y}}{\alpha_{y}+\theta_{y}} \cdot\left(\alpha_{y}+\theta_{y}\right)=\alpha_{y}-\theta_{y} .
$$

Thus, there exist $k:=N+K$ and $z:=\frac{1}{2} x \oplus \frac{1}{2} T^{N} x \in C$ such that for all $m \geq k$, $d\left(z, T^{m} y\right) \leq \alpha_{y}-\theta_{y}$. This means that $\alpha_{y}-\theta_{y} \in A_{y}$. Since $\alpha_{y}-\theta_{y}<\alpha_{y}=\inf A_{y}$, we have got a contradiction.

It follows that $x$ is a fixed point of $T$.

\section{A general logical metatheorem}

One of the main results of this paper, Theorem 3.5 , is a quantitative version of an asymptotic regularity theorem for asymptotically nonexpansive mappings of hyperbolic spaces. In this section we indicate how such a version can be obtained from a prima-facie ineffectively proven convergence result by means of a general logical metatheorem. Such metatheorems were developed first in [13] and [4] and guarantee for general classes of theorems and proofs the extractability of uniform effective bounds from given proofs (see [15] for a comprehensive treatment). The metatheorems apply to general classes of spaces such as metric, hyperbolic, normed, uniformly convex and inner product spaces (as well as their completions) and functions such as nonexpansive, Lipschitz, weakly quasinonexpansive or uniformly continuous functions, among others. We state here only one particular corollary of such a metatheorem which covers the situation treated in this paper.

The formal system $\mathcal{A}^{\omega}[X, d, W]_{-b}$ results from the extension of a system $\mathcal{A}^{\omega}$ for analysis (going back to Spector [26]) obtained by axiomatizing an abstract hyperbolic space $(X, d, W)$. This is achieved by adding constants $d_{X}$ and $W_{X}$ representing $d, W$ to the system together with axioms expressing that $d_{X}$ is a pseudo-metric and $W_{X}$ satisfies the axioms (W1)-(W4) (the subscript ' $-b$ ' refers to the fact that we do not assume $(X, d, W)$ to be bounded). Equality for objects in $X$ is defined as $x={ }_{X} y: \equiv$ $d_{X}(x, y)=\mathbb{R} \quad 0$ so that we actually consider the metric space induced by the pseudometric $d_{X}$. The language of $\mathcal{A}^{\omega}[X, d, W]_{-b}$ is based on the language of functionals of all types over $\mathbb{N}, X$ together with appropriate induction and recursion axioms as well as the axiom schema of dependent choice for all types (which, in particular, implies countable choice and-as a consequence-full comprehension over natural numbers). So in particular full so-called 2 nd order arithmetic is a subsystem of $\mathcal{A}^{\omega}$. Precise definitions for all 
this can be found in [13, 4]. To have quantifiers for functionals over $\mathbb{N}, X$ means that we can quantify not only over $\mathbb{N}$ (starting from 0 ) and $X$ but also over functions $f: \mathbb{N} \rightarrow \mathbb{N}$, $g: X \rightarrow X, h: \mathbb{N} \rightarrow X$ (i.e. sequences in $X$ ) and even over function(al)s taking such objects as arguments and so on. The types $\mathbb{N}, \mathbb{N} \rightarrow \mathbb{N}$ (and also $k$-ary number-theoretic functions), $X, X \rightarrow X, \mathbb{N} \rightarrow X$ are called small types. If we treat general Polish (i.e. complete separable) metric spaces $P$ as continuous images of the Baire space $\mathbb{N}^{\mathbb{N}}$, the type $\mathbb{N} \rightarrow \mathbb{N}$ also covers quantification over $P$ (for Polish spaces $P$ given in so-called standard representation). $\mathcal{A}^{\omega}[X, d, W, \eta]_{-b}$ results from $\mathcal{A}^{\omega}[X, d, W]_{-b}$ by adding a new constant $\eta: \mathbb{N} \times \mathbb{N} \rightarrow \mathbb{N}$ together with axioms expressing that $\eta$ represents a modulus of uniform convexity of $(X, d, W)$ (see [19]). $A_{\forall}$ (resp. $B_{\exists}$ ) is called a $\forall$-formula (resp. an $\exists$-formula) if it has the form $\forall \underline{a} A_{\mathrm{qf}}(\underline{a})$ (resp. $\exists \underline{a} B_{\mathrm{qf}}(\underline{a})$ ) where $\underline{a}$ is a tuple of variables of small types and $A_{\mathrm{qf}}$ (resp. $\left.B_{\mathrm{qf}}\right)$ is a quantifier-free formula.

Let $A_{\forall}(x, y, z, T, u)$ and $B_{\exists}(x, y, z, T, v)$ be $\forall$ - resp. $\exists$-formulas which only contain the shown variables as free variables. In the following we abbreviate $A_{\forall}(x, y, z, T, u)$ and $B_{\exists}(x, y, z, T, v)$ by $A_{\forall}$ and $B_{\exists}$. For $T: X \rightarrow X, x \in X$ and $b \in \mathbb{N}$, the formula Fix $_{\varepsilon}(T, x, b) \neq \emptyset$ expresses that $T$ has an $\varepsilon$-fixed point $p$ in the $b$-ball around $x$, i.e. $d(x, p) \leq b$ and $d(p, T(p))<\varepsilon$.

Theorem 5.1. Let $\left(\lambda_{n}\right)$ be some standard enumeration of $\mathbb{Q}_{+}^{*}$.

1. ([4, Corollary 4.26]) Let P (resp. K) be an $\mathcal{A}^{\omega}$-definable Polish space (resp. a compact metric space). Assume one can prove in $\mathcal{A}^{\omega}[X, d, W]_{-b}$ a sentence

$$
\begin{aligned}
\forall x \in P \forall y \in K \forall n \in \mathbb{N} \forall z \in X \forall T: X \rightarrow X \\
\quad\left(T \lambda_{n} \text {-Lipschitz } \wedge \operatorname{Fix}(T) \neq \emptyset \wedge \forall u \in \mathbb{N} A_{\forall} \rightarrow \exists v \in \mathbb{N} B_{\exists}\right) .
\end{aligned}
$$

Then from the proof one can extract a computable $\oint^{1}$ functional $\Phi: \mathbb{N}^{\mathbb{N}} \times \mathbb{N} \times \mathbb{N} \rightarrow \mathbb{N}$ such that for all representatives $r_{x} \in \mathbb{N}^{\mathbb{N}}$ of $x \in P$ and all $n, b \in \mathbb{N}$,

$$
\begin{array}{r}
\forall y \in K \forall z \in X \forall T: X \rightarrow X\left(T \lambda_{n} \text {-Lipschitz } \wedge \forall \varepsilon>0 \operatorname{Fix}_{\varepsilon}(T, x, b) \neq \emptyset\right. \\
\left.\wedge d_{X}(z, T(z)) \leq \mathbb{R} b \wedge \forall u \leq \Phi\left(r_{x}, n, b\right) A_{\forall} \rightarrow \exists v \leq \Phi\left(r_{x}, n, b\right) B_{\exists}\right)
\end{array}
$$

in all (nonempty) hyperbolic spaces $(X, d, W)$.

2. ([19]) If the premise of this rule is proved in $\mathcal{A}^{\omega}[X, d, W, \eta]_{-b}$, then the conclusion holds in all (nonempty) uniformly convex hyperbolic spaces $(X, d, W)$ provided that $\eta$ is interpreted to be a modulus of uniform convexity of $(X, d, W)$. The bound $\Phi$ then additionally depends on $\eta$.

For the special cases where $P$ is $\mathbb{N}$ with the discrete metric resp. $\mathbb{N}^{\mathbb{N}}$ with the product metric (Baire space), we can treat the elements of $P$ directly without any representation, i.e. $r_{x} \equiv x$. Instead of a single universal premise $B_{\forall}$ we may have a finite conjunction of such premises. Instead of one space $P$ and one space $K$ we may have tuples of (potentially different) such spaces.

The main features of Theorem 5.1 are the following:

\footnotetext{
1 Here we refer to the usual oracle version ('type-2') of computability when dealing with arguments in $\mathbb{N}^{\mathbb{N}}$.
} 
- The extractability of a computable bound on both the premise and the conclusion (of course in practice these bounds will be different, but by taking their maximum one can always obtain a common bound which makes things easier to state). In any concrete case, the bound extracted will not only be computable but of (usually low) subrecursive complexity depending on the principles used in the proof at hand. In our case we will obtain a rather simple bound in the end.

- The bound is highly uniform as it does not depend on $y \in K$, and on $x, T$ and $(X, d, W)$ only via an upper bound $b$ on $d(z, T(z))$ and the distance of (approximate) fixed points of $T$ from $z$ (plus $\eta$ in the case of $\mathcal{A}^{\omega}[X, d, W, \eta]_{-b}$ ).

- The assumption that $T$ has a fixed point is replaced by the existence of approximate fixed points (in some ball around $z$ ). The latter is usually more elementary to verify than the former and does not require the completeness of $X$ or closedness of $C$ (see Section 3).

The following theorem (essentially based on [23, 24, 25]) is proved in [16, Corollary 8] for the case of uniformly convex Banach spaces but its proof can be generalized to uniformly convex hyperbolic spaces.

Theorem 5.2. Let $(X, d, W)$ be a (nonempty) uniformly convex hyperbolic space having a monotone modulus of uniform convexity $\eta, C \subseteq X$ be a nonempty convex subset and $T: C \rightarrow C$ be asymptotically nonexpansive with sequence $\left(k_{n}\right) \in[0, \infty)^{\mathbb{N}}$ where $\sum_{i=0}^{\infty} k_{i}<\infty$. Let $\left(\lambda_{n}\right)$ be a sequence in $[a, b]$ for $0<a<b<1$. If T has a fixed point, then $T$ is $\lambda_{n}$-asymptotically regular.

Since any convex subset of a hyperbolic space is again a hyperbolic space, it suffices to consider only functions $T: X \rightarrow X$. Then Theorem 5.2 can be formalized as follows:

$$
\begin{aligned}
& \forall K, L, k \in \mathbb{N} \forall\left(\lambda_{n}\right) \in[0,1]^{\mathbb{N}} \forall\left(k_{n}\right) \in[0, K]^{\mathbb{N}} \forall x \in X \forall T: X \rightarrow X \\
& \left(\begin{array}{l}
\operatorname{Mon}(\eta) \wedge \forall n \in \mathbb{N} \forall y, z \in X\left(d_{X}\left(T^{n} y, T^{n} z\right) \leq \mathbb{R}\left(1+k_{n}\right) d_{X}(y, z)\right) \\
\wedge \forall n \in \mathbb{N}\left(\sum_{i=0}^{n} k_{i} \leq K\right) \wedge L \geq 2 \wedge \forall n \in \mathbb{N}\left(1 / L \leq \mathbb{R} \lambda_{n} \leq \mathbb{R} 1-1 / L\right) \\
\wedge \operatorname{Fix}(T) \neq \emptyset \\
\rightarrow \exists n \in \mathbb{N} \forall m \in \mathbb{N}\left(d_{X}\left(x_{n+m}, T x_{n+m}\right) \leq \mathbb{R} 2^{-k}\right)
\end{array}\right) .
\end{aligned}
$$

Here, $\operatorname{Mon}(\eta)$ is the $\forall$-formula from [19] expressing that $\eta$ is monotone in the first argument (viewed as a rational number). Then

$$
\begin{aligned}
\operatorname{Mon}(\eta) \wedge & \forall n \in \mathbb{N} \forall y, z \in X\left(d_{X}\left(T^{n} y, T^{n} z\right) \leq \mathbb{R}\left(1+k_{n}\right) d_{X}(y, z)\right) \\
& \wedge \forall n \in \mathbb{N}\left(\sum_{i=0}^{n} k_{i} \leq K\right) \wedge L \geq 2 \wedge \forall n \in \mathbb{N}\left(1 / L \leq \mathbb{R} \lambda_{n} \leq_{\mathbb{R}} 1-1 / L\right)
\end{aligned}
$$

is a finite conjunction of $\forall$-formulas and $[0,1]^{\mathbb{N}},[0, K]^{\mathbb{N}}$ are compact metric (and hence Polish) spaces ( $\mathbb{N}$ is also covered by quantification over $P$ as mentioned above). 
Remark 5.3. Strictly speaking, $[0, K]^{\mathbb{N}}$ is not a single compact metric space but a sequence of such spaces as $K$ varies over $\mathbb{N}$. However, this simple extension is also covered by (the proof of) Theorem 5.1 .

The asymptotic nonexpansivity of $T$,

$$
\forall n \in \mathbb{N} \forall y, z \in X\left(d_{X}\left(T^{n} y, T^{n} z\right) \leq \mathbb{R}\left(1+k_{n}\right) d_{X}(y, z)\right),
$$

implies that $T$ is $\left(1+k_{1}\right)$-Lipschitz continuous. Since $k_{1} \leq K$, in fact $T$ is $(1+K)$ Lipschitz. So we do not need to add a Lipschitz constant as an extra input in order to be able to apply the logical metatheorem.

Unfortunately, the conclusion

$$
\left.\exists n \in \mathbb{N} \forall m \in \mathbb{N}\left(d_{X}\left(x_{n+m}, T x_{n+m}\right)\right) \leq \mathbb{R} 2^{-k}\right)
$$

is not an $\exists$-formula, but only its weakened form

$$
\exists n \in \mathbb{N}\left(d_{X}\left(x_{n}, T x_{n}\right)<\mathbb{R} 2^{-k}\right)
$$

is one.

Suppose now that the proof of Theorem 5.2 can be formalized in $\mathcal{A}^{\omega}[X, d, W, \eta]_{-b}$ (as is the case). Then the logical metatheorem stated above guarantees the extractability of a computable bound $\Phi(K, L, b, \eta, k)$ such that the following holds in all (nonempty) uniformly convex hyperbolic spaces $(X, d, W, \eta)$ with monotone modulus $\eta$ : for all $K$, $L, k, b \in \mathbb{N},\left(\lambda_{n}\right) \in[0,1]^{\mathbb{N}},\left(k_{n}\right) \in[0, K]^{\mathbb{N}}, x \in X, T: X \rightarrow X$ if $T$ is asymptotically nonexpansive with sequence $\left(k_{n}\right), \lambda_{n} \in[1 / L, 1-1 / L]$ for all $n \in \mathbb{N}, \sum_{k=0}^{\infty} k_{n} \leq K$ and

$$
\forall \varepsilon>0\left(\operatorname{Fix}_{\varepsilon}(T, x, b) \neq \emptyset\right) \wedge d(x, T x) \leq b
$$

then

$$
\exists n \leq \Phi(K, L, b, \eta, k)\left(d\left(x_{n}, T x_{n}\right)<2^{-k}\right) .
$$

The original convergence statement

(1) $\forall k \in \mathbb{N} \exists n \in \mathbb{N} \forall m \in \mathbb{N}\left(d\left(x_{n+m}, T x_{n+m}\right)<2^{-k}\right)$

can be rewritten as

(2) $\forall k \in \mathbb{N} \exists n \in \mathbb{N} \forall m \in \mathbb{N} \forall i \in[n, n+m]\left(d\left(x_{i}, T x_{i}\right)<2^{-k}\right)$.

(2) clearly implies the so-called Herbrand normal form $(2)^{H}$ of (2),

$(2)^{H} \forall k \in \mathbb{N} \forall g: \mathbb{N} \rightarrow \mathbb{N} \exists n \in \mathbb{N} \forall i \in[n, n+g(n)]\left(d\left(x_{i}, T x_{i}\right)<2^{-k}\right)$.

Ineffectively, also the converse is true, i.e. (2) ${ }^{H}$ implies (2) (and so also (1)): Assume that $\left(2^{H}\right)$ is true. If (2) were false, then for some $k \in \mathbb{N}$,

$$
\forall n \in \mathbb{N} \exists m_{n} \in \mathbb{N} \exists i \in\left[n, n+m_{n}\right]\left(d\left(x_{i}, T x_{i}\right) \geq 2^{-k}\right) .
$$


Define $g(n):=m_{n}$. Then $\left(2^{H}\right)$ applied to $g$ leads to a contradiction. Due to the ineffectivity of this argument, a bound on ' $\exists n \in \mathbb{N}$ ' in $\left(2^{H}\right)$ cannot be converted effectively into a bound on ' $\exists n \in \mathbb{N}$ ' in (2). Now

$$
\forall i \in[n, n+g(n)]\left(d\left(x_{i}, T x_{i}\right)<2^{-k}\right)
$$

is equivalent to an $\exists$-formula (using that $<$ between real numbers is an existential formula and the universal quantifier over $i$ is bounded). Moreover, quantification over $\mathbb{N}^{\mathbb{N}}$ is covered (as mentioned above) even without any extra representation of the Baire space $\mathbb{N}^{\mathbb{N}}$ as a Polish metric space.

Hence one can apply the logical metatheorem also to the conclusion $\left(2^{H}\right)$ rather than just the special case

$$
\exists n \in \mathbb{N}\left(d\left(x_{n}, T x_{n}\right)<2^{-k}\right),
$$

which corresponds to $g(n) \equiv 0$. As a result we can extract a computable bound $\Phi$ on ' $\exists n \in \mathbb{N}^{\prime}$ ' in $\left(2^{H}\right)$ which in addition to $K, L, b, \eta, k$ also depends on $g$, i.e.

(3) $\exists n \leq \Phi(K, L, b, \eta, k, g) \forall i \in[n, n+g(n)]\left(d\left(x_{i}, T x_{i}\right)<2^{-k}\right)$.

for all $g: \mathbb{N} \rightarrow \mathbb{N}$.

The rest of this paper is concerned with the construction of such a bound $\Phi(K, L, b$, $\eta, k, g$ ), that is, with the proof of Theorem 3.5. We will carry out this construction directly by generalizing the reasoning from [16] rather than first proving Theorem 5.2 and then extracting the bound from the proof. Note, however, that [16] was developed using the extraction algorithm underlying the proof of (earlier versions of) Theorem 5.1 (in its version for uniformly convex normed spaces). As (3) implies (2) ${ }^{H}$ and so (ineffectively) (1) we will obtain Theorem 5.2 as a corollary.

Remark 5.4. At the time the paper [16] was written, the only logical metatheorems available ([13]) required the boundedness of the convex subset in question. Only in [4] could the fact that the results in [16] did not require any global boundedness assumption be accounted for by general logical theorems. In [19] this treatment was adapted to uniformly convex hyperbolic spaces, i.e. the context of the present paper.

\section{Some technical lemmas}

In the following, $(X, d, W)$ is a hyperbolic space, $C \subseteq X$ a nonempty convex subset of $X, T: C \rightarrow C$ an asymptotically nonexpansive mapping with sequence $\left(k_{n}\right),\left(\lambda_{n}\right)$ is a sequence in $[0,1]$ and $\left(x_{n}\right)$ is the Krasnosel'skiı̌-Mann iteration starting with $x \in C$.

Lemma 6.1. Let $n \in \mathbb{N}, p \in C, \alpha>0, \gamma \geq \max \left\{d(T p, p), d\left(T^{n} p, p\right)\right\}$ and $K \geq k_{m}$ for all $m \in \mathbb{N}$. Then

1. $d\left(T^{n} x_{n+1}, x_{n+1}\right) \leq\left(1+k_{n}\right) d\left(T^{n} x_{n}, x_{n}\right)$ and

$$
d\left(x_{n+1}, T x_{n+1}\right) \leq d\left(T^{n+1} x_{n+1}, x_{n+1}\right)+(1+K)^{2} d\left(T^{n} x_{n}, x_{n}\right) .
$$


2. Assume that for both $i=n$ and $i=n+1$ we have

$$
d\left(x_{i}, p\right)<\alpha \quad \text { or } \quad d\left(T^{i} x_{i}, x_{i}\right)<\alpha .
$$

Then

$$
d\left(x_{n+1}, T x_{n+1}\right)<\left(1+(1+K)^{2}(2+K)\right) \alpha+\left(1+K^{2}\right) \gamma
$$

Proof. 1. We have

$$
\begin{aligned}
d\left(T^{n} x_{n+1}, x_{n+1}\right)= & d\left(T^{n} x_{n+1},\left(1-\lambda_{n}\right) x_{n} \oplus \lambda_{n} T^{n} x_{n}\right) \\
\leq & \left(1-\lambda_{n}\right) d\left(T^{n} x_{n+1}, x_{n}\right)+\lambda_{n} d\left(T^{n} x_{n+1}, T^{n} x_{n}\right) \quad(\text { by }(\mathrm{W} 1)) \\
\leq & \left(1-\lambda_{n}\right) d\left(T^{n} x_{n+1}, T^{n} x_{n}\right)+\left(1-\lambda_{n}\right) d\left(T^{n} x_{n}, x_{n}\right) \\
& +\lambda_{n} d\left(T^{n} x_{n+1}, T^{n} x_{n}\right) \\
= & d\left(T^{n} x_{n+1}, T^{n} x_{n}\right)+\left(1-\lambda_{n}\right) d\left(T^{n} x_{n}, x_{n}\right) \\
\leq & \left(1+k_{n}\right) d\left(x_{n+1}, x_{n}\right)+\left(1-\lambda_{n}\right) d\left(T^{n} x_{n}, x_{n}\right) \\
= & \left.\left(1+k_{n}\right) \lambda_{n} d\left(T^{n} x_{n}, x_{n}\right)+\left(1-\lambda_{n}\right) d\left(T^{n} x_{n}, x_{n}\right) \quad \text { (by (1) }\right) \\
\leq & \left(1+k_{n}\right) d\left(T^{n} x_{n}, x_{n}\right), \\
d\left(x_{n+1}, T x_{n+1}\right) \leq & d\left(x_{n+1}, T^{n+1} x_{n+1}\right)+d\left(T^{n+1} x_{n+1}, T x_{n+1}\right) \\
\leq & d\left(x_{n+1}, T^{n+1} x_{n+1}\right)+\left(1+k_{1}\right) d\left(T^{n} x_{n+1}, x_{n+1}\right) \\
\leq & d\left(x_{n+1}, T^{n+1} x_{n+1}\right)+\left(1+k_{1}\right)\left(1+k_{n}\right) d\left(T^{n} x_{n}, x_{n}\right) \\
\leq & d\left(x_{n+1}, T^{n+1} x_{n+1}\right)+(1+K)^{2} d\left(T^{n} x_{n}, x_{n}\right) .
\end{aligned}
$$

2. We have the following cases:

- $d\left(x_{n+1}, p\right)<\alpha$. Then

$$
\begin{aligned}
d\left(x_{n+1}, T x_{n+1}\right) & \leq d\left(x_{n+1}, p\right)+d(p, T p)+d\left(T x_{n+1}, T p\right) \\
& \leq d\left(x_{n+1}, p\right)+\left(1+k_{1}\right) d\left(x_{n+1}, p\right)+d(p, T p) \\
& =\left(2+k_{1}\right) d\left(x_{n+1}, p\right)+d(T p, p)<(2+K) \alpha+\gamma .
\end{aligned}
$$

- $d\left(x_{n+1}, p\right) \geq \alpha$. Then we must have $d\left(T^{n+1} x_{n+1}, x_{n+1}\right)<\alpha$. We distinguish two situations:

(a) $d\left(T^{n} x_{n}, x_{n}\right)<\alpha$. Then, by [19],

$$
d\left(x_{n+1}, T x_{n+1}\right) \leq d\left(T^{n+1} x_{n+1}, x_{n+1}\right)+(1+K)^{2} d\left(T^{n} x_{n}, x_{n}\right)<\left(1+(1+K)^{2}\right) \alpha .
$$

(b) $d\left(x_{n}, p\right)<\alpha$. Then

$$
\begin{aligned}
d\left(T^{n} x_{n}, x_{n}\right) & \leq d\left(T^{n} x_{n}, T^{n} p\right)+d\left(T^{n} p, p\right)+d\left(x_{n}, p\right) \\
& \leq\left(2+k_{n}\right) d\left(x_{n}, p\right)+d\left(T^{n} p, p\right)<(2+K) \alpha+\gamma .
\end{aligned}
$$

Hence, again by (19),

$$
\begin{aligned}
d\left(x_{n+1}, T x_{n+1}\right) & \leq d\left(T^{n+1} x_{n+1}, x_{n+1}\right)+(1+K)^{2} d\left(T^{n} x_{n}, x_{n}\right) \\
& <\alpha+(1+K)^{2}((2+K) \alpha+\gamma) \\
& \leq\left(1+(1+K)^{2}(2+K)\right) \alpha+\left(1+K^{2}\right) \gamma .
\end{aligned}
$$


Lemma 6.2. Let $(X, d, W)$ be a uniformly convex hyperbolic space with a monotone modulus of uniform convexity $\eta$. Let $x, p \in C$ and $K \geq k_{m}$ for all $m \in \mathbb{N}$. Assume that $n \in \mathbb{N}, \alpha, \beta, \beta^{*}, \tilde{\beta}, \gamma, v>0$ are such that

$$
d\left(T^{n} p, p\right)<v \leq 1, \quad \alpha \leq d\left(x_{n}, p\right) \leq \beta, \tilde{\beta}, \beta^{*} \quad \text { and } \quad \alpha \leq d\left(x_{n}, T^{n} x_{n}\right) .
$$

Then

$$
d\left(x_{n+1}, p\right)<d\left(x_{n}, p\right)+k_{n} \beta^{*}+v-2 \alpha \lambda_{n}\left(1-\lambda_{n}\right) \eta\left((1+K) \tilde{\beta}+1, \frac{\alpha}{(1+K) \beta+1}\right) \text {. }
$$

If, moreover, $\eta$ can be written as $\eta(r, \varepsilon)=\varepsilon \cdot \tilde{\eta}(r, \varepsilon)$ where $\tilde{\eta}$ increases with $\varepsilon$ (for a fixed $r$ ), then

$$
d\left(x_{n+1}, p\right)<d\left(x_{n}, p\right)+k_{n} \beta^{*}+v-2 \alpha \lambda_{n}\left(1-\lambda_{n}\right) \tilde{\eta}\left((1+K) \tilde{\beta}+1, \frac{\alpha}{(1+K) \beta+1}\right) .
$$

Proof. Let

$$
r:=\left(1+k_{n}\right) d\left(x_{n}, p\right)+d\left(T^{n} p, p\right), \quad \varepsilon:=\frac{\alpha}{(1+K) \beta+1} \quad \text { and } \quad \psi:=\frac{\alpha}{r} .
$$

By hypothesis, $r<(1+K) \beta+1$, hence $0<\varepsilon<\psi \leq 1$.

We note that

$$
\begin{aligned}
d\left(T^{n} x_{n}, p\right) & \leq d\left(T^{n} x_{n}, T^{n} p\right)+d\left(T^{n} p, p\right) \leq r \\
d\left(x_{n}, p\right) & \leq r \\
d\left(x_{n}, T^{n} x_{n}\right) & \geq \alpha=r \psi \geq r \varepsilon .
\end{aligned}
$$

We get

$$
\begin{aligned}
d\left(x_{n+1}, p\right) & =d\left(\left(1-\lambda_{n}\right) x_{n} \oplus \lambda_{n} T^{n} x_{n}, p\right) \\
& \leq\left(1-2 \lambda_{n}\left(1-\lambda_{n}\right) \eta(r, \varepsilon)\right) \cdot r \quad(\text { by Lemma 2.11 }) \\
& \leq\left(1-2 \lambda_{n}\left(1-\lambda_{n}\right) \eta((1+K) \tilde{\beta}+1, \varepsilon)\right) \cdot r \\
& \quad \quad(\text { since } r<(1+K) \tilde{\beta}+1 \text { and } \eta \text { is monotone }) \\
& =r-2 r \lambda_{n}\left(1-\lambda_{n}\right) \eta((1+K) \tilde{\beta}+1, \varepsilon) \\
& \leq r-2 \alpha \lambda_{n}\left(1-\lambda_{n}\right) \eta((1+K) \tilde{\beta}+1, \varepsilon) \quad(\text { since } r \geq \alpha) \\
& <d\left(x_{n}, p\right)+k_{n} \beta^{*}+v-2 \alpha \lambda_{n}\left(1-\lambda_{n}\right) \eta((1+K) \tilde{\beta}+1, \varepsilon) .
\end{aligned}
$$

Assume now that $\eta(r, \varepsilon)=\varepsilon \cdot \tilde{\eta}(r, \varepsilon)$ and $\tilde{\eta}$ increases with $\varepsilon$. Applying again Lemma 2.11 and the monotonicity of $\eta$, but with $\psi$ instead of $\varepsilon$, we obtain

$$
\begin{aligned}
d\left(x_{n+1}, p\right) & \leq\left(1-2 \lambda_{n}\left(1-\lambda_{n}\right) \eta((1+K) \tilde{\beta}+1, \psi)\right) \cdot r \\
& =\left(1-2 \lambda_{n}\left(1-\lambda_{n}\right) \psi \tilde{\eta}((1+K) \tilde{\beta}+1, \psi)\right) \cdot r \\
& =r-2 \alpha \lambda_{n}\left(1-\lambda_{n}\right) \tilde{\eta}((1+K) \tilde{\beta}+1, \psi) \\
& \leq r-2 \alpha \lambda_{n}\left(1-\lambda_{n}\right) \tilde{\eta}((1+K) \tilde{\beta}+1, \varepsilon) \quad(\text { since } \varepsilon<\psi) \\
& <d\left(x_{n}, p\right)+k_{n} \beta^{*}+v-2 \alpha \lambda_{n}\left(1-\lambda_{n}\right) \tilde{\eta}((1+K) \tilde{\beta}+1, \varepsilon) .
\end{aligned}
$$

We shall also use the following quantitative lemmas on sequences of real numbers. 
Lemma 6.3. Let $\left(a_{n}\right)_{n \geq 0}$ be a real sequence. Then

$$
\forall \varepsilon>0 \forall g: \mathbb{N} \rightarrow \mathbb{N}\left(a_{g^{M}(0)} \geq 0 \rightarrow \exists i<M\left(a_{g^{i}(0)}-a_{g^{i+1}(0)} \leq \varepsilon\right)\right),
$$

where $M:=\left\lceil a_{0} / \varepsilon\right\rceil$. As a consequence,

$$
\forall \varepsilon>0 \forall g: \mathbb{N} \rightarrow \mathbb{N}\left(\forall n \leq \Theta\left(a_{0}, \varepsilon, g\right)\left(a_{n} \geq 0\right) \rightarrow \exists N \leq \Theta\left(a_{N}-a_{g(N)} \leq \varepsilon\right)\right),
$$

where $\Theta\left(a_{0}, \varepsilon, g\right):=\max \left\{g^{i}(0): i \leq M\right\}$. Moreover, $N=g^{i}(0)$ for some $i<M$.

Proof. Let $\varepsilon>0$ and $g: \mathbb{N} \rightarrow \mathbb{N}$ be such that $a_{g M(0)} \geq 0$. Assume by contradiction that $a_{g^{i}(0)}-a_{g^{i+1}(0)}>\varepsilon$ for all $i \in \overline{0, M-1}$. By adding these inequalities, we get $a_{0}-a_{g^{M}(0)}>M \varepsilon=\left\lceil a_{0} / \varepsilon\right\rceil \cdot \varepsilon \geq\left(a_{0} / \varepsilon\right) \cdot \varepsilon=a_{0}$, hence $a_{0}-a_{g^{M}(0)}>a_{0}$, which is a contradiction, since $a_{g^{M}(0)} \geq 0$.

The following lemma is a special case of [16, Lemma 17] $]^{2}$

Proposition 6.4. Let $A_{1}, A_{2} \geq 1, B_{1}, B_{2}, C_{1}, C_{2} \geq 0$ and define, for any $\theta>0$ and any $g: \mathbb{N} \rightarrow \mathbb{N}$,

$$
\Psi\left(A_{1}, A_{2}, B_{1}, B_{2}, C_{1}, C_{2}, g, \theta\right):=h^{M}(0),
$$

where

$$
\begin{aligned}
& h(n):=g(n)+n, \quad D_{i}:=\left(A_{i}+C_{i}\right) \exp \left(B_{i}\right), \\
& M:=\left\lceil 3\left(4 B_{1} D_{1}+4 C_{1}+D_{1}+4 B_{2} D_{2}+4 C_{2}+D_{2}\right) / \theta\right\rceil .
\end{aligned}
$$

Let $\left(a_{n}\right),\left(b_{n}\right),\left(c_{n}\right),\left(\alpha_{n}\right),\left(\beta_{n}\right),\left(\gamma_{n}\right)$ be real sequences such that for all $n \leq \Psi$,

$$
a_{n}, b_{n}, c_{n}, \alpha_{n}, \beta_{n}, \gamma_{n} \geq 0, \quad a_{n+1} \leq\left(1+b_{n}\right) a_{n}+c_{n}, \quad \alpha_{n+1} \leq\left(1+\beta_{n}\right) \alpha_{n}+\gamma_{n},
$$

and moreover

$a_{0} \leq A_{1}, \quad \alpha_{0} \leq A_{2}, \quad \sum_{n=0}^{\Psi} b_{n} \leq B_{1}, \quad \sum_{n=0}^{\Psi} \beta_{n} \leq B_{2}, \quad \sum_{n=0}^{\Psi} c_{n} \leq C_{1}, \quad \sum_{n=0}^{\Psi} \gamma_{n} \leq C_{2}$.

Then the following holds:

1. $a_{n} \leq D_{1}, \alpha_{n} \leq D_{2}$ for all $n \leq \Psi+1$;

2. for all $\theta \in(0,1]$ and all $g: \mathbb{N} \rightarrow \mathbb{N}$,

$$
\exists N \leq \Psi \forall i, j \in[N, N+g(N)]\left(\left|a_{j}-a_{i}\right| \leq \theta \wedge\left|\alpha_{j}-\alpha_{i}\right| \leq \theta\right) .
$$

Moreover, $N=h^{i}(0)$ for some $i<M$.

\footnotetext{
2 Corrections to [16]: In Lemma 15 and later, $\lfloor\cdot\rfloor$ should be $\left\lceil\cdot 7\right.$. P. 164, line $4:{ }^{\prime} j \leq m+g(m)$ ' should be ' $j \leq m+g(m)+1$ ', and consequently, in Theorem 22 and Corollary 28 , ' $h=\lambda n .(g(n+$ 1) $+n+1$ ' should be ' $h=\lambda n .\left(g(n+1)+n+2\right.$ ', and in Corollary 25, ' $n \leq \Phi_{1}$ ' must be replaced by ' $n \leq 2 \Phi_{1}$ '.
} 


\section{Proof of Theorem 3.5}

Let $\varepsilon \in(0,1]$ and $g: \mathbb{N} \rightarrow \mathbb{N}$ be arbitrary and $K, L, x \in C, b, h: \mathbb{N} \rightarrow \mathbb{N}, M, D, \theta$, $f(K), \Phi$ be as given in the hypotheses of Theorem 3.5 Let us remark that

$$
\frac{\varepsilon}{f(K)((1+K) D+1)}<\frac{1}{2}<1,
$$

and moreover $\theta \leq \varepsilon /\left(L^{2} f(K)\right)<1$.

Since $x \in C$ and $b>0$ satisfy (4), there exists $p \in C$ such that

$$
d(x, p) \leq b \quad \text { and } \quad d(p, T p) \leq \frac{1}{2^{\Phi}(\Phi+K)} .
$$

Since

$$
d\left(T^{n} p, p\right) \leq d\left(T^{n} p, T^{n-1} p\right)+d\left(T^{n-1} p, p\right) \leq\left(1+k_{n-1}\right) d(p, T p)+d\left(T^{n-1} p, p\right),
$$

it follows that for all $1 \leq n \leq \Phi$,

$$
\begin{aligned}
d\left(T^{n} p, p\right) & \leq \sum_{i=0}^{n-1}\left(1+k_{i}\right) d(p, T p)=d(p, T p)\left(n+\sum_{i=0}^{n-1} k_{i}\right) \\
& \leq(n+K) \cdot \frac{1}{2^{\Phi}(\Phi+K)} \leq \frac{1}{2^{\Phi}} \leq \frac{1}{2^{n}} .
\end{aligned}
$$

Let us consider the sequences

$a_{n}:=d\left(x_{n}, p\right), \quad \alpha_{0}:=K D+2, \quad \alpha_{n}:=K D+2-\sum_{i=0}^{n-1}\left(k_{i} D+\frac{1}{2^{i}}\right) \quad$ for $n \geq 1$.

Then for all $n \leq \Phi$, we have $0 \leq \alpha_{n+1} \leq \alpha_{n}$ and

$$
\begin{aligned}
0 \leq a_{n+1} & =d\left(\left(1-\lambda_{n}\right) x_{n} \oplus \lambda_{n} T^{n} x_{n}, p\right) \leq\left(1-\lambda_{n}\right) d\left(x_{n}, p\right)+\lambda_{n} d\left(T^{n} x_{n}, p\right) \\
& \leq\left(1-\lambda_{n}\right) d\left(x_{n}, p\right)+\lambda_{n} d\left(T^{n} x_{n}, T^{n} p\right)+\lambda_{n} d\left(T^{n} p, p\right) \\
& \leq\left(1-\lambda_{n}\right) d\left(x_{n}, p\right)+\lambda_{n}\left(1+k_{n}\right) d\left(x_{n}, p\right)+\lambda_{n} d\left(T^{n} p, p\right) \\
& \leq\left(1+k_{n}\right) d\left(x_{n}, p\right)+d\left(T^{n} p, p\right) \leq\left(1+k_{n}\right) a_{n}+1 / 2^{n} .
\end{aligned}
$$

It is easy to verify that we can apply Proposition 6.4 with $a_{n}, \alpha_{n}$ given as above, $b_{n}:=k_{n}$, $c_{n}:=1 / 2^{n}, \beta_{n}:=\gamma_{n}:=0, A_{1}:=b, B_{1}:=K, C_{1}:=2, A_{2}:=K D+2, B_{2}:=C_{2}:=0$, $\tilde{g}(n):=g(n+1)+2$ and $\theta, \Phi$ as above.

It follows by Proposition 6.4 that

$$
a_{n} \leq\left(A_{1}+C_{1}\right) \exp \left(B_{1}\right)=D \quad \text { for all } n \leq \Phi
$$

and that there exists $N_{0} \leq \Phi$ with $N_{0}=h^{s}(0)$ for some $s<M$ such that

$$
\forall i, j \in\left[N_{0}, N_{0}+g\left(N_{0}+1\right)+2\right]\left(\left|a_{j}-a_{i}\right| \leq \theta \wedge\left|\alpha_{j}-\alpha_{i}\right| \leq \theta\right) .
$$


In fact, since the sequence $\left(h^{n}(0)\right)$ is strictly increasing, we have $N_{0}=h^{s}(0)<h^{M}(0)$ $=\Phi$, so $N_{0}+1 \leq \Phi$.

Let $N:=N_{0}+1$. In the following, we shall prove that $N$ satisfies (5), that is,

$$
\forall m \in[N, N+g(N)]\left(d\left(x_{m}, T x_{m}\right)<\varepsilon\right) .
$$

Let $m \in[N, N+g(N)]$. Then $m-1, m, m+1 \in\left[N_{0}, N_{0}+g\left(N_{0}+1\right)+2\right]$, so we can apply (27) with $i \in\{m-1, m\}$ and $j=i+1$ to get

$$
\left|d\left(x_{i+1}, p\right)-d\left(x_{i}, p\right)\right|=\left|a_{i+1}-a_{i}\right| \leq \theta \quad \text { and } \quad k_{i} D+1 / 2^{i}=\left|\alpha_{i+1}-\alpha_{i}\right| \leq \theta .
$$

Moreover,

$$
m-1<m \leq N_{0}+1+g\left(N_{0}+1\right)<h\left(N_{0}\right)=h^{s+1}(0) \leq h^{M}(0) \leq \Phi .
$$

Let $i \in\{m-1, m\}$ and assume that $d\left(x_{i}, p\right) \geq \varepsilon / f(K)$ and $d\left(T^{i} x_{i}, x_{i}\right) \geq \varepsilon / f(K)$. Then

$$
d\left(T^{i} p, p\right) \leq \frac{1}{2^{\Phi}}<\frac{1}{2^{i}} \leq 1, \quad \frac{\varepsilon}{f(K)} \leq d\left(T^{i} x_{i}, x_{i}\right), \quad \frac{\varepsilon}{f(K)} \leq d\left(x_{i}, p\right) \leq D
$$

(by (26)), so we can apply Lemma 6.2, (20) with $\alpha:=\varepsilon / f(K), v:=1 / 2^{i}, \beta:=\beta^{*}:=$ $\tilde{\beta}:=D$, the definition of $\theta$ and the fact that $\lambda_{i}\left(1-\lambda_{i}\right) \geq 1 / L^{2}$ to get

$$
d\left(x_{i+1}, p\right)<d\left(x_{i}, p\right)+k_{i} D+1 / 2^{i}-2 \theta .
$$

It follows that

$$
2 \theta<d\left(x_{i}, p\right)-d\left(x_{i+1}, p\right)+k_{i} D+1 / 2^{i}=a_{i}-a_{i+1}+k_{i} D+1 / 2^{i} \leq 2 \theta,
$$

by 28$]$, which is a contradiction.

Hence, for both $i=m$ and $i=m-1$,

$$
d\left(x_{i}, p\right)<\varepsilon / f(K) \quad \text { or } \quad d\left(T^{i} x_{i}, x_{i}\right)<\varepsilon / f(K) .
$$
that

Finally, applying Lemma 6.12 with $n:=m-1, \alpha:=\varepsilon / f(K), \gamma:=1 / 2^{\Phi}$ it follows

$$
\begin{aligned}
d\left(x_{m}, T x_{m}\right) & <\left(1+(1+K)^{2}(2+K)\right) \frac{\varepsilon}{f(K)}+\left(1+K^{2}\right) \frac{1}{2^{\Phi}} \\
& =\frac{\varepsilon}{2}+(1+K)^{2} \frac{1}{2^{\Phi}} \quad(\text { by the definition of } f(K)) \\
& <\frac{\varepsilon}{2}+\left(1+K^{2}\right) \frac{1}{2^{m}} \quad(\text { since } m<\Phi), \\
& \leq \frac{\varepsilon}{2}+(1+K)^{2} \theta \quad\left(\text { since } 1 / 2^{m} \leq \theta, \text { by }[28)\right. \\
& <\varepsilon
\end{aligned}
$$

since $(1+K)^{2} \theta \leq(1+K)^{2} \varepsilon / L^{2} f(K)<\varepsilon / 2$.

Acknowledgments. The research reported in this paper was carried out during the authors' stay at the Max-Planck-Institute for Mathematics (Bonn) whose support is gratefully acknowledged. 


\section{References}

[1] Borwein, J., Reich, S., Shafrir, I.: Krasnosel'ski-Mann iterations in normed spaces. Canad. Math. Bull. 35, 21-28 (1992) Zbl 0712.47050 MR 1157459

[2] Bridson, M., Haefliger, A.: Metric Spaces of Non-Positive Curvature. Grundlehren Math. Wiss. 319, Springer, Berlin (1999) Zbl 0988.53001 MR 1744486

[3] Clarkson, J. A.: Uniformly convex spaces. Trans. Amer. Math. Soc. 40, 396-414 (1936) Zbl 0015.35604 MR 1501880

[4] Gerhardy, P., Kohlenbach, U.: General logical metatheorems for functional analysis. Trans. Amer. Math. Soc. 360, 2615-2660 (2008) Zbl 1130.03036 MR 2373327

[5] Goebel, K.: Concise Course on Fixed Point Theory. Yokohama Publ., Yokohama (2002) Zbl 1066.47055 MR 1996163

[6] Goebel, K., Kirk, W. A.: A fixed point theorem for asymptotically nonexpansive mappings. Proc. Amer. Math. Soc. 35, 171-174 (1972) Zbl 0256.47045 MR 0298500

[7] Goebel, K., Kirk, W. A.: Iteration processes for nonexpansive mappings. In: S. P. Singh et al. (eds.), Topological Methods in Nonlinear Functional Analysis (Toronto, 1982), Contemp. Math. 21, Amer. Math. Soc., Providence, RI, 115-123 (1983) Zbl 0525.47040 MR 0729507

[8] Goebel, K., Reich, S.: Uniform Convexity, Hyperbolic Geometry, and Nonexpansive Mappings. Monogr. Textbooks Pure Appl. Math. 83, Dekker, New York (1984) Zbl 0537.46001 MR 0744194

[9] Khamsi, M. A.: On asymptotically nonexpansive mappings in hyperconvex metric spaces. Proc. Amer. Math. Soc. 132, 365-373 (2004) Zbl 1043.47040 MR 2022357

[10] Kirk, W. A.: Krasnosel'skii iteration process in hyperbolic spaces, Numer. Funct. Anal. Optim. 4, 371-381 (1982) Zbl 0505.47046 MR 0673318

[11] Kirk, W. A.: Geodesic geometry and fixed point theory II. In: J. García Falset et al. (eds.), International Conference on Fixed Point Theory and Applications (Valencia, 2003), Yokohama Publ., Yokohama, 113-142 (2004) Zbl 1083.53061 MR 2144169

[12] Kohlenbach, U.: Analysing proofs in analysis. In: W. Hodges et al. (eds.), Logic: from Foundations to Applications (Staffordshire, 1993), Oxford Sci. Publ., Oxford Univ. Press, New York, 225-260 (1996) Zbl 0881.03032 MR 1428007

[13] Kohlenbach, U.: Some logical metatheorems with applications in functional analysis. Trans. Amer. Math. Soc. 357, 89-128 (2005) Zbl 1079.03046 MR 2098088

[14] Kohlenbach, U.: Effective uniform bounds from proofs in abstract functional analysis. In: B. Cooper et al. (eds.), New Computational Paradigms: Changing Conceptions of What is Computable, Springer, Berlin, 223-258 (2008) Zbl pre05269063

[15] Kohlenbach, U.: Applied Proof Theory: Proof Interpretations and their Use in Mathematics. Springer Monogr. Math.. Springer, Berlin (2008) Zbl pre05248785 MR 2445721

[16] Kohlenbach, U., Lambov, B.: Bounds on iterations of asymptotically quasi-nonexpansive mappings. In: J. García Falset et al. (eds.), International Conference on Fixed Point Theory and Applications (Valencia, 2003), Yokohama Publ., Yokohama, 143-172 (2004) Zbl 1089.47053 MR 2144170

[17] Kreisel, G.: On the interpretation of non-finitist proofs, part I. J. Symbolic Logic 16, 241-267 (1951) Zbl 0044.00302 MR 0049135

[18] Leuştean, L.: A quadratic rate of asymptotic regularity for CAT(0)-spaces. J. Math. Anal. Appl. 325, 386-399 (2007) Zbl 1103.03057 MR 2273533

[19] Leuştean, L.: Proof mining in $\mathbb{R}$-trees and hyperbolic spaces. In: G. Mints and R. de Queiroz (eds.), Proceedings of the 13th Workshop on Logic, Language, Information and Computation (WoLLIC 2006) (Stanford, 2006), Electron. Notes Theor. Comput. Sci. 165, Elsevier, Amsterdam, 95-106 (2006) MR 2321766 
[20] Liu, Q.: Iteration sequences for asymptotically quasi-nonexpansive mappings with error member. J. Math. Anal. Appl. 259, 18-24 (2001) Zbl 1001.47034 MR 1836441

[21] Liu, Q.: Iteration sequences for asymptotically quasi-nonexpansive mapping with an error member of uniform convex Banach space. J. Math. Anal. Appl. 266, 468-471 (2002) Zbl 1057.47057 MR 1880518

[22] Reich, S., Shafrir, I.: Nonexpansive iterations in hyperbolic spaces. Nonlinear Anal. 15, 537558 (1990) Zbl 0728.47043 MR 1072312

[23] Rhoades, B. E.: Fixed point iterations for certain nonlinear mappings. J. Math. Anal. Appl. 183, 118-120 (1994) Zbl 0807.47045 MR 1273436

[24] Schu, J.: Iterative construction of fixed points of asymptotically nonexpansive mappings. J. Math. Anal. Appl. 158, 407-413 (1991) Zbl 0734.47036 MR 1117571

[25] Schu, J.: Weak and strong convergence to fixed points of asymptotically nonexpansive mappings. Bull. Austral. Math. Soc. 43, 153-159 (1991) Zbl 0709.47051 MR 1086729

[26] Spector, C.: Provably recursive functionals of analysis: a consistency proof of analysis by an extension of principles formulated in current intuitionistic mathematics. In: J. C. E. Dekker (ed.), Proc. Sympos. Pure Math. 5, Amer. Math. Soc., Providence, RI, 1-27 (1962) Zbl 0143.25502 MR 0154801

[27] Takahashi, W.: A convexity in metric space and nonexpansive mappings, I. Kōdai Math. Sem. Rep. 22, 142-149 (1970) Zbl 0268.54048 MR 0267565

[28] Tao, T.: Soft analysis, hard analysis, and the finite convergence principle. Essay posted May 23, 2007. Also appeared in: Tao, T., Structure and Randomness: Pages from Year One of a Mathematical Blog, Amer. Math. Soc. (2008)

[29] Tao, T.: Norm convergence of multiple ergodic averages for commuting transformations. Ergodic Theory Dynam. Systems 28, 657-688 (2008) Zbl pre05272920 MR 2408398 